\title{
EL TRATAMIENTO INFORMATIVO Y PUBLICITARIO DE LOS DIARIOS DIGITALES ESPECIALIZADOS EN GOLF EN ESPAÑA
}

\author{
José Gabriel Fernández. Fernández. \\ Esther Martínez Pastor \\ Universidad Rey Juan Carlos. España
}

\section{RESUMEN}

El espectacular desarrollo del golf en los últimos 20 años en España ha popularizado este deporte ampliando su función deportiva, económica y social, apoyándose en el sector turístico. Esta realidad ha provocado también un lógico interés por los medios de comunicación especializados en golf, y en la última década han surgido numerosas cabeceras digitales sobre esta industria con contenidos informativos y publicitarios tanto deportivos como de un estilo de vida característico. En este artículo se analizan esos contenidos y se estudian sus peculiaridades.

Palabras clave: Golf; turismo; diarios digitales; información; publicidad.

\section{Information and advertising of digital newspapers specialized in golf in Spain}

\section{ABSTRACT}

The spectacular development of golf in the past 20 years in Spain has popularized this sport expanding its sporting, economic and social function, with strong support in the tourism sector. This reality has also led to a logical interest in specialized media in golf, and in the last decade numerous digital newspapers on this industry have emerged with information and advertising about sports and a lifestyle characteristic. This paper analyzes these contents and studies his peculiarities.

Keywords: Golf; tourism; digital newspapers; information; advertising.

Recibido: 7 de agosto de 2014

Devuelto para su revisión: 11 de diciembre de 2014

Aceptado: 3 de febrero de 2015

Facultad de Ciencias de la Comunicación. Universidad Rey Juan Carlos. Camino del Molino, s/n. Campus de Fuenlabrada. 28943 MADRID (España).E-mail: josegabriel.fernandez@urjc.es, esther.martinez.pastor@urjc.es 


\section{GOLF, TURISMO Y MEDIOS DE COMUNICACIÓN}

El golf en España ha desarrollado en los últimos 20 años un espectacular ascenso de aficionados, si en 1990 había 45.000 jugadores en España, a finales de 2013 la cifra alcanzaba los 296.000, según la Real Federación Española de Golf (2013). Este aumento de jugadores ha sucedido también en otros países como Estados Unidos que contaba con 3,5 millones de jugadores en 1950 y en 2000 pasó a tener 26 millones (Geissler, 2005). Esta evolución en el número de practicantes ha provocado un fuerte desarrollo del sector, que además de tener un alto componente deportivo, tanto profesional como amateur, está muy relacionado con el turismo y con un estilo de vida que involucra a otros sectores como la gastronomía, la salud o el medio ambiente, y combina a la perfección con actividades empresariales y de marketing en un mundo cada vez más profesionalizado (Castro, 2009, De la Torre, 2002). De hecho, a nivel mundial se estima que aproximadamente las ganancias derivadas del turismo de golf son de diez mil millones de dólares, de los cuales ocho mil millones corresponden a Estados Unidos y el resto a Europa (a, Paniza, 2005).

El turismo en España es uno de los soportes de su economía, con un peso del $11 \%$ de su PIB en 2012 (INE, 2012). Parece, por tanto, que la apuesta por el turismo debe seguir y el golf es uno de los atractivos para este importante sector. Junto al tradicional sol y playa de España, el golf es un reclamo al turismo (Feo Parrondo, 2001), y además desestacionalizado, es decir, que si en el verano predomina el turista de playa, en invierno acude el de golf (Riquel y Vargas, 2012; Martínez, 1986 y Priesley y Sabi, 1987). Este es el caso de Andalucía (Martínez Pastor y Nicolás, 2014), Turquía (Çuhadar, 2013) o Colorado (Wilson y Thilmany, 2006), entre otros, que venden como uno de sus atractivos turísticos los campos de golf.

La consultora Aguirre Newman, en un estudio de 2011, afirmaba que a España acuden al año cerca de un millón de turistas de golf, siendo el segundo país en recibir turistas de golf por detrás de Estados Unidos. Cada uno de ellos se gasta una media de 170 euros al día aproximadamente, y visitan nuestro país una semana de media, lo que supone unos ingresos totales de alrededor de 1.200 millones de euros al año (Aguirre Newman; 2011). La segmentación de este público es esencial como parte del turismo dado que cabe diferenciar el perfil de los profesionales al de los aficionados que pueden ser apasionados, habituales, ocasionales o interesados (Lyu, y Lee, 2013; Malgorn, 1996). Esto indica que el espectacular desarrollo del golf en los últimos 20 años en España ha popularizado este deporte ampliando su función deportiva, económica y social, apoyándose en el sector turístico.

En todo caso, todavía sigue siendo un deporte con numerosos obstáculos, prejuicios y estereotipos, como que es un deporte de élite, de clase social alta, de hombres y contrario al medio ambiente (Espejo Marín, 2004; Mass y Hasbrook, 2001). Paniza (2005) recoge del estudio de Amorós (2002) un perfil del turista de golf en España, que suele ser mayoritariamente un jugador mayor de 50 años $(56,2 \%)$, mujer en un 14,24\%, y con una escasa presencia de jóvenes $(3,9 \%$ ) (Amorós, 2002). Además, otro informe de Deporte \& Business de 2006, publicado en por la Real Federación Española de Golf (2006), asegura que el perfil de jugador de golf en España es de un hombre mayor de 35 años (85\%), 
casado (80\%), con dos hijos (64\%), propietario de un vehículo Premium (50\%), directivo o empresario (50\%), y profesional liberal (75\%), mientras que un $20 \%$ de las mujeres jugadoras de golf no trabaja, y un $28 \%$ es directiva o empresaria.

En ese sentido, el Libro Blanco del Golf en Madrid, editado por la Federación Madrileña de Golf (2011), asegura que un 10\% de los jugadores de golf en Madrid son de clase alta.

El binomio de turismo y golf, de economía y deporte, ha sido identificado como un nuevo nicho de mercado y de información con un target determinado. Esto ha dado lugar a la aparición de nuevas cabeceras digitales especializadas en este deporte (Fernández, J. G. 2014), con una publicidad segmentada adecuada para este público. Desde 2005 han ido apareciendo diferentes blogs y diarios dedicados al golf, cabeceras digitales con información diaria sobre este deporte y todo su ámbito. Sin embargo desde el punto de vista periodístico, la multiplicación estos diarios digitales no implica mayor especialización periodística dado que en ocasiones los autores se alejan de la audiencia convirtiéndose en un experto más, olvidando su función periodística y divulgativa (Fernández del Moral, J. 2004). Y desde el punto de vista publicitario, el exceso de estereotipos en los anuncios pueden distorsionar y alejarse de la realidad causando rechazo en el público potencial y objetivo (Mass y Hasbrook, 2001).

Por ello, nos centramos como punto de partida en el trabajo realizado por Frideres y Palao (2006). Llevaron a cabo un estudio comparado entre dos cabeceras digitales elmundo.es y usatoday.com, de España y USA, con el objetivo de analizar las noticias relativas al deporte para saber cómo y qué interesa comunicar del deporte en los diarios de mayor difusión de sendos países. Se abordaron las temáticas de las noticias, las noticias deportivas en función del nivel de los deportistas (equipos nacionales, internacionales y universitarios), en relación al género y al tipo de deporte. Entre las conclusiones más relevantes identificaron que son pocas las noticias relativas a la práctica deportiva, que el género masculino tiene la mayor presencia frente a una escasa aparición de la mujer y que son cuatro los deportes que monopolizan las noticias deportivas (baloncesto, fútbol, fútbol americano y béisbol), siendo el golf el séptimo deporte abordado por estas cabeceras nacionales. En cuanto a la publicidad, según Hernández (2008), la imagen estereotipada es un campo de golf cerca de un Parque Natural que evoca la naturaleza y siempre vinculado a un slogan green que conjugue la práctica del golf con el paisaje natural. Generalmente se publicitan actividades que integran este binomio como hoteles, centro termales, playas, etc., en donde el jugador pueda descansar y disfrutar (Hernández, 2008).

Al hilo de estos trabajos, este estudio pretende identificar y reflejar el estado de los principales diarios digitales especializados en golf en España para analizar el tratamiento informativo y publicitario de éstos. El propósito es dual: de un lado, constatar si esos mensajes se ajustan a una correcta información especializada y si la publicidad representa a su target, y de otro lado, reflexionar si los contenidos informativos y los publicitarios, al unísono, reflejan la misma realidad del colectivo de jugadores de golf, tal y como se han hecho en otras investigaciones relativas a la relación entre el periodismo y la publicidad (Santibáñez, y Vergara, 2008) o a determinados colectivos como inmigrantes (Martínez Pastor y Santín, 2009) o del sector del entretenimiento (Grandi, 1995). 


\section{OBJETIVOS E HIPÓTESIS DE ESTUDIO}

El principal objetivo de este estudio es identificar las principales cabeceras de diarios digitales relacionadas con el golf y establecer una clasificación de los temas que más interesan a estas cabeceras digitales, resolviendo cuáles son las temáticas que más se publican en el ámbito del golf por los diarios especializados, así como conocer de las marcas, los productos y servicios anunciados en estas cabeceras y las representaciones de los anuncios, siguiendo el estudio de Frideres y Palao (2006).

Los objetivos secundarios de la investigación pretenden establecer el grado de opinión frente a la información con el que cuentan los diarios estudiados y, a un mismo tiempo, identificar si la publicidad representa las necesidades del público objetivo, de acuerdo con los estudios analizados, y si se podrían identificar nuevos nichos de mercado, según el Informe del Real Federación Española de Golf (2006) y del Libro Blanco del Golf en Madrid (2011).

Otro objetivo conocer si el golf como deporte y como actividad turística construye unos estereotipos que pueden provocar prejuicios. Esta actividad se ha relacionado casi siempre con un público del alto nivel adquisitivo, reducido, elitista y cerrado. La evolución de los aficionados al golf ha podido cambiar estas peculiaridades y la información de golf es un interesante testigo de este cambio, al hilo del trabajo de Frideres y Palao (2006).

Para lograr estos objetivos que proponen estas hipótesis:

H1: Los diarios digitales especializados en golf hacen un seguimiento periodístico de esta industria desde un punto de vista deportivo, económico y social.

H2: La publicidad que está en los diarios digitales sobre golf está construida con estereotipos que generan una imagen de este deporte que no se ajusta a la realidad.

H3: La información y la publicidad de los diarios digitales especializados en golf no reflejan la misma realidad. Cada una de las comunicaciones representa una realidad del golf diferente.

\section{METODOLOGÍA Y PROCEDIMIENTO}

El universo de la muestra han sido los principales diarios digitales especializados en golf. Para ello, se han elegido aquellos que cumplan con unos claros requisitos para identificarlos como cibermedios. En primer lugar el predominio de la información como contenido periodístico, una característica clave para diferenciarse de un blog, una web institucional o una simple distribuidora de noticias que cuente con enlaces a otros sitios web (Alonso, 2010). En segundo lugar la actualización, un criterio que permite establecer más concretamente la actividad periodística de estos cibermedios. En tercer lugar, se han tenido en cuenta las cabeceras digitales que muestren una clara vocación multimedia, de hipertextualidad y de interactividad, cualidades imprescindibles para ser reconocido como cibermedios (Alonso y Martínez, 2003). En cuarto lugar, como criterio numérico para establecer una clasificación lógica que pueda seleccionar estas cabeceras digitales, se han elegido las que cuenten con más de 500 seguidores en Twitter, un número significativo para determinar el seguimiento de estos diarios digitales en las redes sociales. Por último, 
que en los sites hubiera diferentes modalidades de publicidad como comercial, autopromoción y patrocinios. De este modo la muestra de diarios digitales que se han analizado son los siguientes:

- Ten-golf.es con 4.484 seguidores el 27 de marzo de 2014.

- Cronicagolf.com con 3.728 seguidores el 27 de marzo de 2014

- Lapaginadelgolf.es con 2.702 seguidores el 27 de marzo de 2014

- Opengolf.com con 1.804 seguidores el 27 de marzo de 2014

- Elperiodigolf.com con 1.640 seguidores el 27 de marzo de 2014

- Golfconfidencial.com con 813 seguidores el 27 de marzo de 2014

- Doblebogey.es (Golfcast) con 909 seguidores el 27 de marzo de 2014

El lapso de tiempo analizado ha sido desde el 1 de febrero de 2014 hasta el 28 de marzo de 2014, tanto a las 12.00 horas como a las 18.00 horas cada día. Estos dos meses se sitúan en medio de la temporada profesional de golf y en el comienzo de las actividades amateurs más importantes como la Feria de Golf MadridGolf o los Circuitos de golf para aficionados más representativos. El hecho de revisar el diario digital dos veces al día se debe a poder constatar mejor la actualización de información.

Una vez fijados los criterios de selección de los diarios digitales sobre golf, se explican ahora los parámetros de estudio, primero con un criterio cualitativo y después con un criterio cuantitativo, contabilizando, de un lado, el número de noticias en portada clasificadas por temas, y después divididas por el tipo de elaboración de la información, por la opinión, y por el uso del multimedia y, de otro, la representación que se hace del golf a través de la publicidad.

En cuanto al análisis cualitativo, el número de noticias se ha clasificado según sus temas, estableciendo las categorías siguientes:

- Profesional: Informaciones relativas a la competición profesional que incluye las diferentes pruebas profesionales por todo el mundo.

- Amateur: Informaciones referentes a la competición amateur como son competiciones de los equipos nacionales y territoriales de la Real Federación Española de Golf.

- Sector: Iniciativas de empresas relacionadas con el golf, actividades y novedades acerca de la industria del golf.

- Comercial: Comunicaciones de ofertas de diferentes empresas o del propio diario.

- Torneos: Pruebas de aficionados organizadas por empresas.

- Campos de golf: Información relativa a los campos de golf.

- Salud: Información relativa a la salud en el deporte del golf.

- Viajes: Información vinculada al turismo de golf.

- Gastronomía: Información acerca de restaurantes y bodegas.

- Medio Ambiente: Información relacionada con la sostenibilidad.

Por su parte, la categorización que se ha hecho para identificar la construcción del contenido publicitario ha sido a través de la siguiente división: el anunciante, el género 
representado, categoría de producto, acciones y objetos representados y modalidad publicitaria, tal y como se han hecho en trabajos similares en otros sectores (Martínez, Nicolás, Salas: 2013 y Moreno, 2003). Se trata de elaborar un informe acerca del tipo de información y publicidad con la que cuentan estas cabeceras, y por eso se han estudiado además los siguientes criterios. Primero el número de informaciones propias, es decir, firmadas por la redacción o un colaborador, frente a las informaciones que provienen de las notas de prensa de organizaciones o de empresas, calificadas como no propias. En segundo lugar se ha estudiado el número de vídeos que publican los diarios digitales, comprobando así la importancia multimedia de este tipo de cibermedios, tanto si son de elaboración propia como si proceden de cualquier otro canal. También se ha diferenciado entre información y opinión, a partir del número de piezas informativas y piezas argumentativas.

En cuanto al análisis cuantitativo, se ha elaborado una ficha para cuantificar los ítems anteriormente descritos relativos a la información y a la publicidad, llegando a computarse más de 53 variables, entre las que destacan la temática de las noticias; si son informaciones elaboradas por la reacción o son notas de prensa elaboradas por los distintos campos de golf o empresas del sector; si existen opiniones acerca de la industria del golf y del turismo; si aparecen informaciones multimedia que dan más valor a los contenidos; si la publicidad coincide con los estereotipos del jugador de golf; y en definitiva, si estas cabeceras suponen o no un impulso al conocimiento del golf en España y por lo tanto podrían animar al sector del Turismo (Tabla 1).

\section{Tabla 1 \\ INVESTIGACIÓN CUANTITATIVA}

\begin{tabular}{|l|l|}
\hline Investigación cuantitativa & \\
\hline Universo de la muestra & $\begin{array}{l}\text { Ten-golf.es, } \\
\text { Cronicagolf.com, Lapaginadelgolf.es, Opengolf.com, } \\
\text { Elperiodigolf.com, Golfconfidencial.com, Doblebogey.es }\end{array}$ \\
\hline Muestra & 2190 noticias y anuncios 89 anuncios \\
\hline Periodo & 1 de febrero al 28 de marzo de 2014 \\
\hline Variables analizadas & 53 \\
\hline
\end{tabular}

Fuente: elaboración propia.

\section{RESULTADOS}

Se han podido comprobar diversos resultados que exponemos a continuación en relación al total de las noticias, de las diferentes secciones, del tipo de elaboración de las noticias, de la opinión y de los vídeos en todas cabeceras digitales, así como el total de la publicidad. Además se ha analizado el porcentaje que cada uno de los diarios analizados dedica a cada apartado. A lo largo de los dos meses de estudio se han contabilizado un total de 2.190 noticias entre los siete diarios digitales especializados en golf seleccionados, de las cuales 576 han sido durante los fines de semana, un $26.3 \%$ del total. 


\subsection{Resultados totales}

En cuanto a los totales, sobre el total de noticias, Elperiodigolf es el que más piezas ha publicado con un total de 554, lo que supone un 25.2\% del total, mientras que Doblebogey es el que menos ha publicado con 111 noticias, un 5\% por ciento del total (Gráfico 1).

\section{Gráfico 1 \\ TOTAL DE NOTICIAS}

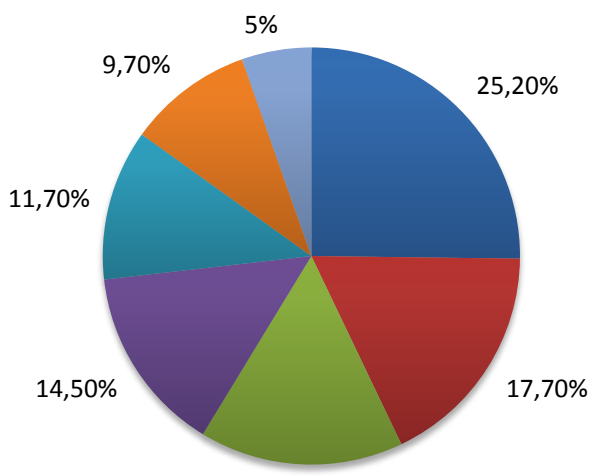

$15,80 \%$
Elperiodigolf.com

- Cronicagolf.com

-Tengolf.com

-Golfconfidencial.com

-Opengolf.com

- Lapaginadelgolf.es

Doblebogey.es

Fuente: elaboración propia.

Por secciones, las noticias que tratan del mundo profesional son, con claridad, las que más aparecen en estos diarios digitales. En total son 1.640 noticias relacionadas con los profesionales de golf y las competiciones profesionales de los diferentes Circuitos por todo el mundo. Esta cifra supone un $75 \%$ del total de noticias. Le siguen, a mucha distancia, las noticias amateurs, competiciones de los equipos nacionales y territoriales de la Real Federación Española de Golf, que cuentan con un seguimiento de 186 noticias, un 8.5\% del total. En lo que respecta a las noticias sobre el sector del golf, con actividades, iniciativas o novedades acerca del mundo del golf, los diarios digitales especializados en golf han publicado en estos dos meses 115 noticias, un 5.2\% del total, un porcentaje similar al de noticias patrocinadas, tanto de auto referencia como de novedades comerciales, con 113 , un $5.1 \%$ del total. Por su parte, los torneos para aficionados organizados por empresas han sido seguidos en 87 ocasiones, lo que supone un $4 \%$ del total. Y en cuanto a campos de golf, en 39 ocasiones se ha publicado alguna noticia, un $1.7 \%$ del total. Más residual son las noticias sobre salud, con 8 noticias, un $0.40 \%$ del total, o las noticias sobre viajes, 2 noticias, un $0.09 \%$ del total. En este período estudiado no ha habido noticias sobre medio ambiente ni sobre gastronomía (Gráfico 2).

En cuanto a los totales por el tipo de elaboración, opinión y vídeos, de todas las piezas publicadas por estos siete diarios digitales, 1.337 han sido de elaboración propia, es decir un $61 \%$ del total, mientras que 566 han sido de elaboración no propia, lo que supone un 


\section{Gráfico 2 \\ TOTALES POR SECCIONES}

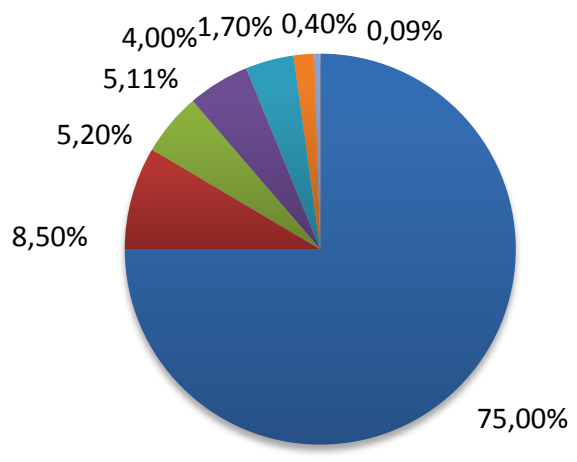

$$
\begin{aligned}
& \text { PROF. } \\
& \text { AMAT. } \\
& \text { SECTOR } \\
& \text { COMER. } \\
& \text { CAMNEO } \\
& \text { SALUD } \\
& \text { VIAJES }
\end{aligned}
$$

Fuente: elaboración propia.

$25.8 \%$ del total. En cuanto a la opinión, se han contabilizado 73 piezas argumentativas, un $3.3 \%$ del total, mientras que en lo que respecta a la capacidad multimedia, en estos dos meses se han publicado 274 vídeos, un 9.8\% del total. De esos vídeos, 42 han sido de elaboración propia, es decir un 19.6\% de los vídeos publicados (Ver Gráfico 3).

\section{Grafico 3 \\ TOTALES POR TIPO DE ELABORACIÓN, OPINIÓN Y VÍDEOS}

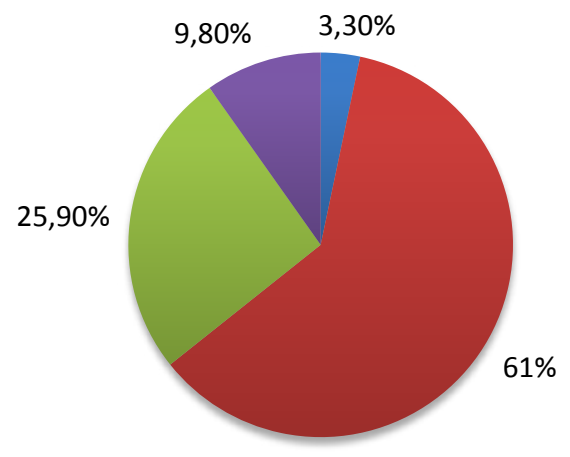

$$
\begin{aligned}
& \text {-OPINION } \\
& \text { - PROPIA } \\
& \text { NOPROP. } \\
& \text { - VIDEOS }
\end{aligned}
$$

Fuente: elaboración propia.

En cuanto a la publicidad los sectores que más se anuncian son los campos de golf con un 26,8\% de presencia; seguido por los medios de comunicación con un $19,5 \%$ y de material deportivo $9,8 \%$. Mientras que los sectores menos representativos en estas cabeceras son eventos, construcciones, grandes almacenes, seguros, textil, restauración, SPA y alquiler de coches con un 1,2\%. Los anunciantes más representativos en las cabeceras ana- 
lizadas, con un índice de aparición del 3,7\%, son Decathlon, los Ángeles de San Rafael, El Encín Golf, Galletas Bahlsen, Turismo de Andorra, Elperiodigolf y Kernpharma, aunque el porcentaje más alto, con un $45,1 \%$, corresponde a sectores varios. En cuanto al género representado en los anuncios prevalece el masculino, con un 19,5\% frente al femenino que tiene una presencia del 4,9\%, de una forma muy similar al estudio de Frideres y Palao (2006). Es habitual encontrar piezas publicitarias sin ningún género representado, con un $73,2 \%$ y sólo en un 2,4\% de los casos aparecen los dos géneros en los anuncios. Las acciones representadas son el juego (16,3\%) y la diversión $(5,8 \%)$, y en un $77,9 \%$ no hay acciones representadas dado que se enfatiza en un campo de golf o en un producto.

En cuanto a los objetos representados y vinculados con el golf los más destacados en los anuncios son palos de golf (28\%), el jugador en el campo de golf $(13,4 \%)$, las bolas de golf $(7,3 \%)$ y las instalaciones $(4,9 \%)$. En cuanto a las modalidades publicitarias más recurrentes en los diarios digitales estudiados son la publicidad comercial con un $84,4 \%$ seguida de promociones $(6,7 \%)$, y de la autopromoción $(6,7 \%)$ y en menor medida, con un $1,1 \%$ de los publirreportajes.

\subsection{Resultados por diarios}

En un análisis por diarios, encontramos que en las noticias profesionales Elperiodigolf es el que más ha publicado con 382 piezas de las 1.640 totales, un $23.2 \%$, mientras que Doblebogey es el que menos con 72 , un $4.4 \%$.

Respecto a las noticias amateur, el diario que más piezas ha publicado sobre esta sección ha sido Golf Confidencial con 75 , un $40.3 \%$ de las 186, mientras que Ten-Golf es el que menos con 5, un 2.6\%. Sobre el sector de golf de nuevo Elperiodigolf es el diario que más ha atendido esta sección, con 33 noticias, lo que supone un 28.6\% de las 115 piezas publicadas. El que menos ha sido Ten-Golf con 2 noticias, un $1.7 \%$. Respecto a las noticias sobre torneos, Golf Confidencial es el que más ha publicado, con 53 piezas de las 87 totales, lo que supone un $60.9 \%$, mientras que Ten-Golf no ha publicado ninguna en estos dos meses estudiados. Las noticias acerca de los campos de golf han tenido más atención por parte de Elperiodigolf y de Golf Confidencial con 14 piezas cada uno, un $35.8 \%$ de las 39 noticias totales. En el lado contrario Ten-Golf y Lapaginadegolf no han publicado ninguna noticia sobre campos de golf. En cuanto a las noticias de salud, de las 8 piezas contabilizadas en total, Elperiodigolf ha publicado 5, un $62.5 \%$, mientras que no habido ninguna en Cronicagolf, Ten-Golf y Golf Confidencial. De las dos noticias sobre viajes, Golf Confidencial y Open Golf han publicado una cada uno, el 50\% de las piezas sobre viajes en estos dos meses. Finalmente, de las 113 las noticias patrocinadas, Elperiodigolf es el que más ha publicado con 70, un 61.9\%, mientras que Lapaginadegolf y Doble Bogey no han publicado ninguna noticia de esta naturaleza.

En lo que respecta a la opinión, Ten-Golf domina esta clasificación con 43 piezas de opinión, un $58.9 \%$ de las 73 opiniones publicadas. Open Golf no ha publicado ninguna. Elperiodigolf es el diario que más noticias de elaboración propia ha publicado con 298, un 22.2\% de las 1.337, mientras que Doble Bogey es el que menos con 44, un $3.3 \%$. En las noticias de elaboración no propia, Golf Confidencial es el que más piezas muestra con 178 , un $31.4 \%$, mientras que Ten-Golf es el que menos con 18 , un $3.2 \%$. 
Sobre los vídeos, de los 214 publicados, Elperiodigolf ha presentado 68, un $31.7 \%$ del total, mientras que Doble Bogey sólo ha publicado 1, un $0.4 \%$. Entre los vídeos propios, Ten-Golf ha publicado 20 vídeos propios de los 42 que se han publicado en total, lo que supone un $47.6 \%$, mientras que Open Golf, Lapaginadegolf y Dobe Bogey no han presentado vídeos propios.

\subsection{Resultados de cada uno de los diarios}

Analizando el porcentaje que cada uno de los diarios dedica a cada apartado se destaca que las noticias profesionales, Ten-Golf es el que más atención le dedica en su diario a este tema, con un $97.4 \%$ del total de sus piezas, mientras que Golf Confidencial es el que menos con un $41.8 \%$ Se cambian las tornas cuando se trata de hablar de noticias amateur y es Golf Confidencial el que más piezas dedica con un $23.5 \%$ de sus noticias, frente al $1.4 \%$ de Ten-Golf. También sobre el sector del golf es Golf Confidencial el que más porcentaje dedica, con un $8.4 \%$ frente al $0.5 \%$ de Ten-Golf. En cuanto a los torneos, Golf Confidencial publica un $16.6 \%$ de sus piezas a esta sección, mientras que Ten-Golf no publica ninguna pieza sobre este tema.

También sobre campos de golf, Golf Confidencial lidera esta tabla con una dedicación del $4.4 \%$ de sus piezas, frente a Ten-Golf y Lapaginadegolf que no publican ninguna noticia sobre este asunto. Sobre salud, Elperiodigolf y Doble Bogey dedican un $0.9 \%$ de sus piezas a este tema, mientras que CronicaGolf, Ten-Golf y Golf Confidencial no dedican ninguna pieza a este asunto. En la sección de viajes, tanto Golf Confidencial como Open Golf dedican un $0,3 \%$ de sus piezas a los viajes, y los demás no publican noticias sobre esta sección. Finalmente, en las noticias patrocinadas Elperiodigolf es el que más porcentaje dedica a esta sección con un 12.6\%, mientras que Lapaginadegolf y Doble Bogey no dedican ninguna pieza.

La clasificación sobre las piezas de opinión es liderada por Ten-Golf, que es el que más porcentaje dedica a la argumentación con un $12.3 \%$ de su contenido, mientras que Open Golf no publica ninguna opinión. Sobre las noticias de elaboración propia, Lapaginadegolf es la que más porcentaje refleja en sus piezas con un 76\% frente al 39.6\% de Doble Bogey. Mientras, en las noticias de elaboración no propia es Doble Bogey la que más porcentaje presenta con un $58.5 \%$ de su diario frente al $5.1 \%$ de Ten-Golf. Respecto a los vídeos, Open Golf es el que refleja un porcentaje más alto de sus piezas, con un $12.4 \%$, mientras que Doble Bogey ha recurrido al vídeo en un $0.9 \%$ de sus informaciones. Sin embargo en los vídeos propios, en Ten-Golf el 51.2\% de sus vídeos son de elaboración propia, mientras que Open Golf, Lapaginadegolf y Doble Bogey no ofrecen vídeos propios.

\subsection{Resultados de actualización de noticias}

En cuanto a la actualización, Elperiodigolf es el diario que más regularidad presenta a la hora de las actualizaciones a las 12 y a las 18 horas, con una media de 6,25 noticias al día por la mañana, y 3,60 noticias al día por la tarde, mientras que Doble Bogey es el que menos noticias nuevas presenta en cada hora de análisis, con una media de 1,51 noticias cada día por la mañana y de 0,46 noticias cada día por la tarde (Gráfico 4). 


\section{ACTUALIZACIÓN DE LAS NOTICIAS}
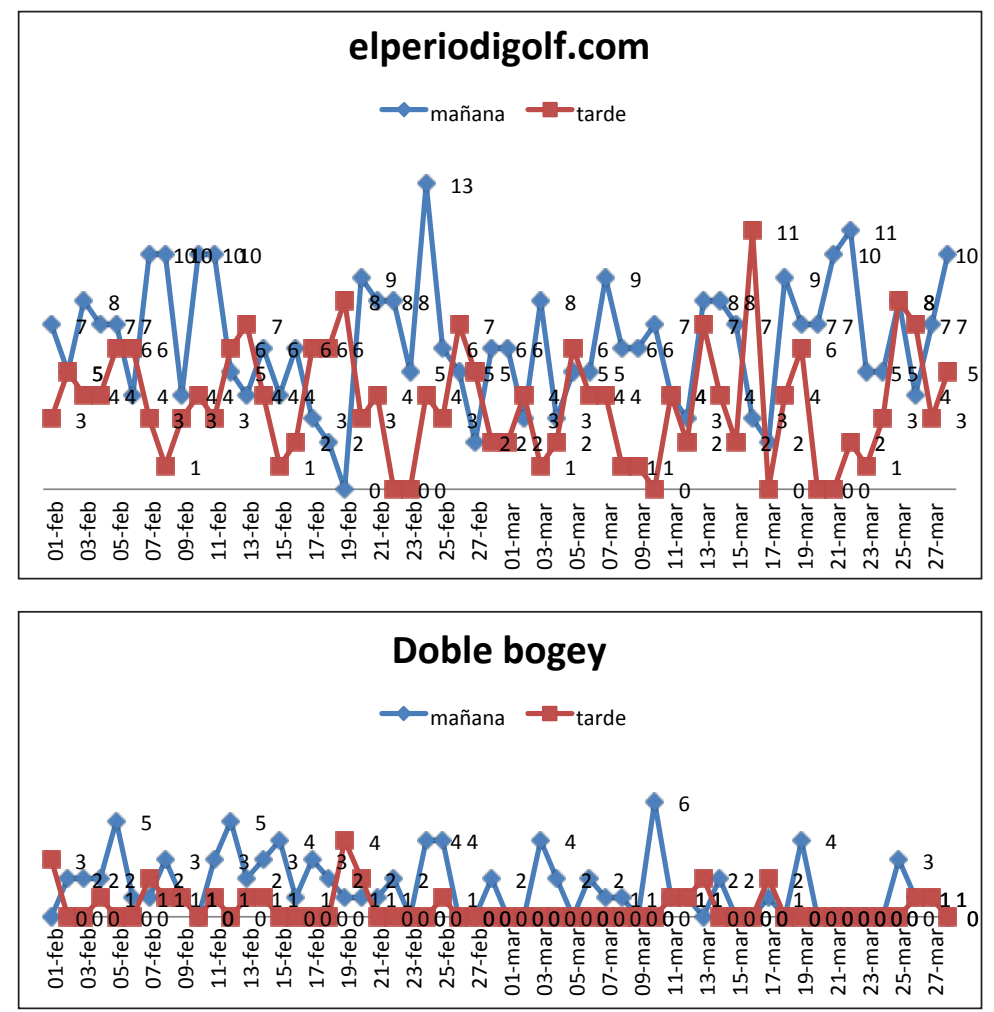

Fuente: elaboración propia.

\section{DISCUSIÓN}

H1: Los diarios digitales especializados en golf hacen un seguimiento periodístico de esta industria desde un punto de vista deportivo, económico y social.

La rápida y espectacular evolución de aficionados al golf en los últimos 20 años, la enorme vinculación de este deporte con el turismo, las numerosas actividades empresariales relacionadas con el golf como instrumento de márketing, y los éxitos deportivos de jugadores profesionales de reconocimiento mundial, han provocado un intenso interés periodístico por esta materia.

Por su parte, la crisis económica y las nuevas tecnologías han acelerado la tendencia cada vez más clara hacia un periodismo digital de los medios de comunicación, y así lo demuestra el aumento de las audiencias de los diarios en la red (EGM, mayo 2014). Además, una mayor especialización amplía las posibilidades periodísticas aumentando el rigor y la profesionalidad para conseguir una mejor divulgación del conocimiento, uno de los 
objetivos fundamentales del periodismo especializado, según Fernández de Moral (2004). Todos estos factores, el desarrollo de la industria del golf junto con un nuevo periodismo muy relacionado con las nuevas tecnologías y más especializado, han ayudado a la aparición de numerosos diarios digitales sobre el mundo del golf, y desde 2005 se pueden contabilizar hasta siete que cumplen con los requisitos que debe tener un cibermedio, como la actualización de contenidos periodísticos, la multimedialidad, la interactividad y la hipertextualidad (Alonso, 2010). Estudiadas estas siete cabeceras digitales especializadas en golf se han podido comprobar algunos resultados significativos. El primero y más claro es que la información que ofrecen está, en su gran mayoría, relacionada con las competiciones profesionales, en un 75 por ciento, dejando para un cuarta parte de su contenido el ámbito social, económico y amateur del golf. Es precisamente el mundo profesional lo que centra la atención de los medios digitales especializados en golf. La competición profesional prima sobre cualquier otro tema relacionado con el golf, dando un carácter claro de diario deportivo al margen de cualquier otra temática más social o económica, de modo que a pesar de que el golf es una industria muy vinculada al turismo, a la economía y al deporte amateur, la mayoría de contenidos periodísticos de estos diarios digitales se dedica a las pruebas de los Circuitos Nacionales e Internacionales, y a los grandes jugadores profesionales de todo el mundo. En todo los casos, la notoriedad de grandes jugadores profesionales y torneos de interés mundial como el Grand Slam (Masters de Augusta, Us Open, Open Championship y PGA Championship), las Series Mundiales o la Ryder Cup, acontecimientos de audiencias millonarias, suponen un fuerte reclamo para prensa y aficionados, y es también la continua celebración de estos acontecimientos a lo largo del año la que ayuda a una mayor actualización de los contenidos de estos diarios digitales especializados en golf. De esta manera, Elperiodigolf presenta una media diaria de 9,85 noticias en el periodo estudiado, frente a las 1,97 de Doblebogey. Sin embargo, además de esta amplia presencia de la competición profesional, estos diarios cuentan con una mirada hacia el carácter económico y social de la industria del golf, con informaciones y opiniones sobre estos ámbitos, intentando abarcar todos los aspectos del golf partiendo, eso sí, de una información deportiva de alto nivel. En este sentido, una mayor diversificación de la información ayudaría a establecer un mayor conocimiento del mundo del golf como industria, ampliando y actualizando con mayor frecuencia tanto los contenidos informativos como los publicitarios. Eso no quiere decir que la actualidad del mundo profesional deje de atenderse, sino más bien que esa información, que sirve muchas veces como soporte a una mayor actualización, pueda vincularse todavía más con los aspectos económicos y sociales del golf. Las informaciones sobre el golf profesional poco pueden influir en el turismo, a menos que sea una competición que se desarrolle en España y eso ayude a promocionar el destino.

Junto a este predominio de las noticias profesionales, llama la atención también la poca presencia de piezas argumentativas en estos diarios digitales especializados en golf, con sólo un 3,3\% del total, así como un minoritario uso del multimedia, con un 9,7\% del total, y además, sólo un 19,6\% de esos vídeos es de elaboración propia. La multimedialidad es una de las características de los diarios digitales, que deben contar cada vez más con apoyo audiovisual en esta nueva era del periodismo (Masip, Díaz, Domingo, Micó-Sanz y Salaverría, 2010; Cabrera, 2010). Por otra parte, un periodismo especializado debe contar 
con un componente mayor de opinión, como dice Fernández del Moral (2004), fruto del mayor conocimiento de los periodistas y de su función de servicio para una mayor divulgación. En cualquier caso, el hecho de que el $61 \%$ de las informaciones sean de elaboración propia sí ayuda a conseguir esa labor periodística.

\section{H2: La publicidad que está en los diarios digitales sobre golf está construida con estereotipos que generan una imagen del golf que no se ajusta a la realidad.}

La publicidad en los diarios digitales muestra un claro estereotipo en cuanto al género de quienes lo practican. Los hombres aparecen en un 19,5\% frente a la escasa presencia de las mujeres, con un 4,9\%. Tal y como afirmaba Mass y Hasbrook, (2001) esto puede generar un estereotipo al asociar este deporte con el género masculino. Sin embargo, si nos remitimos a los datos de Paniza (2005) el segmento de edad del turista de golf en España es un hombre mayor de 50 años $(56,2 \%)$ frente a la mujer que representa sólo un $14,24 \%$. Esto indicaría que la publicidad se dirige realmente a su público dado que el masculino es mayor que el femenino, y no genera diferencias de género sino que se ajusta a su target, que en definitiva es quien les comprará su producto o servicio. Incluso hay anuncios en los que se representa un grupo de jugadores y ninguno de jugadoras. Es un juego claramente de hombres. Sin embargo, la presencia conjunta de ambos géneros con un escaso 2,4\% podría aumentarse para vincular este deporte a la pareja, como de hecho se practica en muchos casos. En cuanto a los estereotipos de que es un deporte vinculado con una clase social alta, los sectores que aparecen de forma repetida en las cabeceras digitales especializadas en golf son viajes $(4,9 \%)$, restauración $(1,2 \%)$, hoteles $(4,9 \%)$, SPA $(1,2 \%)$ o vinos $(3,7 \%)$ aspectos vinculados a un turismo de poder adquisitivo, tal y como indicaba Mass y Hasbrook, (2001). También podemos observar que en la publicidad los valores y acciones representados solamente se vinculan con la diversión, en un 5,8\% mientras que el resto no se centra en ninguna acción. Esto podría cambiar para ofrecer una visión más cercana del golf y asociarlo mucho más con valores de entretenimiento, salud, medio ambiente o deporte familiar. No obstante, el hecho de relacionar el golf con este tipo de segmento social podría ayudar a fomentar un turismo más exclusivo, y la publicidad analizada así lo demuestra.

\section{H3: La información y la publicidad de los diarios digitales especializados en golf no reflejan la misma realidad. Cada una de las comunicaciones representa una imagen del golf diferente.}

La publicidad y el periodismo son dos materias que, aunque se aúnan en los medios de comunicación, tradicionalmente han tenido una difícil relación y de hecho en el Código Deontológico de la Federación de Asociaciones de Periodistas de España se obliga a una separación formal y rigurosa entre la información y la publicidad. Es evidente que sus objetivos son distintos pero que deben cohabitar en un mismo escenario. En el caso de los diarios digitales especializados en golf objeto de este estudio, el mensaje de las informaciones y de los anuncios publicitarios tampoco van de la mano, pero, además, muestran dos realidades diferentes del mundo del golf. 
De un lado está la información que, como hemos visto, centra su atención en el deporte profesional, con amplia presencia de noticias sobre las competiciones nacionales e internacionales y en los jugadores profesionales con un amplio seguimiento por todo el mundo. En este sentido, los diarios digitales especializados en golf españoles presentan unas características claras de medios de información deportiva, y su objetivo principal es informar del golf como deporte, dando respuesta a la demanda de resultados deportivos de carácter profesional y a las actividades de sus estrellas. Así, la audiencia es amplia y heterogénea, ya que lo importante es dar a conocer, por ejemplo, el estado de salud de Tiger Woods, cómo se ha desarrollado el Masters de Augusta, o la gran temporada de Sergio García, al margen de si la audiencia de estos diarios es joven, son turistas, están de vacaciones, son hombres o mujeres. Prima el hecho informativo del mensaje más que el público concreto al que va dirigido, $y$, de hecho, no necesariamente debe ser un público relacionado con el golf. A pesar de todo, para buscar esa cohabitación, la publicidad busca también esa amplia audiencia que dan los grandes eventos deportivos para después centrarse en un público objetivo en cada caso. Por otra parte, el $25 \%$ de la información de estos diarios digitales sí tienen que ver con la industria del golf fuera de los eventos deportivos profesionales, estableciendo pequeños espacios dirigidos a un determinado público que sí estaría más en consonancia con los objetivos publicitarios concretos.

Por su parte, la publicidad que se focaliza en un público objetivo del que conoce sus preferencias y le ofrece productos y servicios asociados a su estilo de vida y al deporte que practica, como son los viajes, el turismo y restauración, entre otros. Se centra en el hombre, que es el que más juega, y menos en la mujer, y sus anuncios se dirigen a los productos necesarios del juego, como son las bolas, los palos o las instalaciones. El espacio en el que se representa el anuncio siempre son espacios abiertos y se focaliza en el color verde del campo de golf, acompañado de agua, ya sea el mar o lagos, para connotar contacto con la naturaleza y tranquilidad. Esto podría cambiarse con una mayor amplitud a un público más joven, dado que según Paniza (2005) hay una presencia de jóvenes jugadores del 3,9\% que está olvidado en la publicidad, así como, potenciar más el juego en pareja. Además, las diferentes empresas interesadas en promocionar sus productos aprovechan, en algunos casos, su relación con deportistas profesionales de golf para que utilicen sus marcas y promocionen sus novedades. De manera que vemos una disonancia entre las noticias, centradas en una información puramente deportiva, frente a los anuncios, que muestran la imagen de un determinado estilo de vida vinculado al golf. La popularización del golf, con un significativo aumento de aficionados en los últimos años, y la fuerte relación de este deporte con el turismo, invita a pensar en un mayor desarrollo tanto de la información como de la publicidad en estos diarios digitales especializados. Por un lado con más atención periodística al mundo del golf como industria, y por otro, con un mejor aprovechamiento publicitario de los grandes acontecimientos deportivos y de las estrellas de este deporte. Así, el turismo de golf podría buscar un mejor impulso en el desarrollo de estas cabeceras especializadas.

\section{CONCLUSIONES}

Los diarios digitales especializados en información sobre golf en España centran su atención en las competiciones profesionales con claridad, con un $75 \%$ del total, superando 
ampliamente otras noticias sobre competición amateur, torneos para aficionados organizados por empresas, el sector del golf, campos de golf, viajes, gastronomía o medio ambiente, y se puede concluir que se da un tratamiento más deportivo que social o económico, unos datos que ayudarían en menor medida a fomentar un turismo de golf. Estos diarios publican en su mayoría noticias de elaboración propia con un $61 \%$ del total, de tal manera que son los propios periodistas especializados los que elaboran las piezas dando un valor añadido a esa información. Mientras, la presencia de la opinión es poco significativa con un 3.3\% del total, y predomina por tanto la información sobre las piezas argumentativas. Es escasa también la multimedialidad, que no está presente de manera clara y sólo un 9,7\% son noticias audiovisuales. Además, de todos los vídeos publicados sólo un 19.6\% son propios. La prensa debería fomentar algo más el periodismo opinión y una mayor utilización de informaciones multimedia que favorezcan la empatía con los lectores.

Por su parte, la publicidad representa claramente al target al que se dirige, hombres de más de 50 años, potenciando valores de una vida de clase alta. Esto podría potenciar más los estereotipos de este deporte, y se podría representar a otros segmentos como los jóvenes, las mujeres o las parejas que, aunque no sean tan numerosos, son un target potencial, así como asociar más el golf con valores vinculados con la educación y la vida saludable más que a un estatus social, tal y como afirman Frideres y Palao (2006). En todo caso, el turismo de golf se puede ver impulsado por este tipo de publicidad potenciando la paridad de género en este deporte y ampliando su nicho de mercado a los jóvenes.

Los mensajes periodísticos y publicitarios en estos diarios digitales de golf reflejan realidades diferentes. Si el contenido periodístico va dirigido a un público amplio y heterogéneo con una gran mayoría de piezas sobre el deporte profesional, el contenido publicitario se centra en un público objetivo determinado, buscando adecuarse al perfil de jugador de golf asociado a un estilo de vida concreto. La evolución del golf como deporte y como industria debería ayudar a profundizar tanto en los contenidos periodísticos, con más información fuera de las competiciones internacionales, como en los contenidos publicitarios, con una mayor vinculación al deporte profesional y ampliando su público objetivo a nuevos aficionados. Esas modificaciones informativas y publicitarias darían, sin duda, más valor al turismo en un sector del golf que busca apoyo en esta importante actividad económica en España.

\section{BIBLIOGRAFÍA}

AGUILÓ PÉREZ, E. (1999): «Consideraciones en torno a los trabajos e investigaciones sobre la calidad en la formación turística», Cuadernos de Turismo , n 4, pp. 7-9.

AGUIRRE NEWMAN (2011): Estudio de mercado. Campos de golf en España. Resumen ejecutivo, La industria del golf en España en http://www.rfegolf.es

ALONSO, J. (2010): «Cibermedios: Conceptualización y tipologías», en Evolución tecnológica y cibermedios. Cabrera González M.A. (Coord.). Zamora, Comunicación Social Ediciones y Publicaciones.

ALONSO, J. y MARTÍNEZ, L. (2003): «Medios Interactivos: Caracterización y contenidos», en Manual de redacción ciberperiodística. Diaz Noci, J. y Salaverría R. (Coord.), Barcelona, Ariel Comunicación. 
DEPORTE \& BUSINESS (2006): Perfil del jugador de golf en España. Disponible en http://www.rfegolf.es/ArtculosDocumento/Turismo\%20e\%20impacto $\% 20$ econ\%C3\%B3mico/informedb07.pdf

CABRERA, M.A. (Coord.) (2010): Evolución tecnológica y cibermedios. Sevilla-Zamora, Comunicación Social.

CASTRO, H. (2009): «Turismo deportivo: importancia actual y futura», Retos Turísticos, Vol. 8 Issue 1, pp. 44-48.

ÇUHADAR MURAT, D. (2013): «Development of golf tourism and golf tourism demand forecasts in Turkey: a study of Belek region», International Journal of Human Sciences, Vol. 10 Issue 1, pp. 1620-1639.

DE LA TORRE, M. (2002): «El marketing de Turespaña como destino de golf», en La diversificación y la desestacionalización del sector turístico. IV Congreso de Turismo Universidad y Empresa. Bigné Alcañiz, J.E. y Blanquer Criado, D. (Dir.), pp. 95-102.

DEPORTE \& BUSINESS (2006): Informe del perfil del jugador habitual de golf en españa. Disponible en http://www.rfegolf.es/ArtculosDocumento/Turismo\%20e\%20 impacto\%20económico/informedb07.pdf

DÍEZ, A., MARTÍN, J.A. y LEGIDO, J. (2011): Libro Blanco del Golf en Madrid. Federación de Golf de Madrid, Madrid.

ESTUDIO GENERAL DE MEDIOS (2014). Disponible en http://www.aimc.es

ESPEJO MARÍN, C. (2004): «Campos de golf y medio ambiente: Una interacción necesaria», Cuadernos de Turismo, n 14, pp. 67-111.

FEO PARRONDO, F. (2001): «Los campos de golf en España y su repercusión en el sector turístico», Cuadernos de Turismo, $\mathrm{n}^{\circ}$ 7, pp. 55-66.

FERNÁNDEZ, J.G. (2011): «Periodismo digital, una ventana a la especialización: El caso de Elperiodigolf.com», en Migración, desarrollo humano e internacionalización. Said, E. (Coord.). colombia, Memorias Cátedra Europa, pp. 379-388.

FERNÁNDEZ, J.G. (2014): «Periodismo especializado en golf en España», en Nuevos retos del periodismo especializado. Esteve Ramírez, F. y Nieto Hernández, J.C. (Coord.). Madrid, Schedas Colección Universidad.

FERNÁNDEZ DEL MORAL, J. (2004): Periodismo especializado. Madrid. Ariel Comunicación.

FRIDERES, J. y PALAO, J. M. (2006): «Análisis de las noticias deportivas de dos periódicos digitales de España y Estados Unidos: ¿promoción de la actividad física y el deporte? », Ciencias aplicadas a la actividad física y el deporte, pp. 7-14. Disponible rn http://redined.mecd.gob.es/xmlui/bitstream/handle/11162/44843/01520123000332. pdf? sequence $=1$

GEISSLER, G. (2005): «An Examination of the Golf Vacation Package-Purchase Decision: A Case Study in the U. S. Golf Coast Region», Journal of Hospitality \& Leisure Marketing, Vol. 13(1), pp. 65-82.

NE (2012): «Producto Interior Bruto regional. Año 2012», Disponible en www.ine.es

HERNÁNDEZ, J. (2008): La imagen de Andalucía en el turismo. Fundación Centro de Estudios Andaluces, Sevilla.

LYU, S. y LEE, H. (2013): «Market Segmentation of Golf Event Spectators Using Leisure Benefits», Journal of Travel \& Tourism Marketing, Vol. 30 Issue 3, pp. 86-200. 
MALGORN, V. (1996): «Le marché britanniqué du golf», en Les cahier espaces, nº 46. MARTÍNEZ, E. (1986): «Presente y futuro del turismo de golf en España», Estudios turísticos, Ministerio de Economía y Hacienda, n. 90, pp. 23-46.

MARTÍNEZ PASTOR, E. y SANTÍN DURÁN, M. (2009): «Publicaciones para inmigrantes: estudio de las informaciones y la publicidad dirigidas a la comunidad inmigrante», Doxa Comunicación: revista interdisciplinar de estudios de comunicación y ciencias sociales, $\mathrm{N}^{\circ}$. 9, 2009, pp. 125-144.

MARTÍNEZ PASTOR, E. y NICOLÁS, M.A. (2013): «La construcción de la imagen de marca Andalucía como destino turístico a través de las campañas 'Smail you are Andalucía' y 'Andalucía te quiere'», Cuadernos de Turismo, n 32, pp. 207-228.

MARTÍNEZ PASTOR, E., NICOLÁS, M.A. Y SALAS, A. (2013): «La representación de género en las campañas de publicidad de juguetes en Navidades (2009-12)», Comunicar, 41, pp. 187-194.

MARTÍNEZ PASTOR, E. y NICOLÁS, M.A. (2014): «The Construction of Tourist Space by Public Administration and Institutional Communication: The Image of the Brand Andalucía as a Tourist Destination», Journal of Promotion Management, 20:2, pp. 181-199.

MASIP, P., DÍAZ, D., DÍAZ, J., DOMINDO, D., MICÓ-SANZ, J. y SALAVERRÍA, R. (2010): «Investigación internacional sobre ciberperiodismo: hipertexto, interactividad, multimedia y convergencia», El profesional de la información, vol. 9, $\mathrm{n}^{\mathrm{o}}$ 6, pp. 568-576.

MASS, K. y HASBROOK, C. (2001): «Media Promotion of the Paradigm Citizen/Golfer: An Analysis of Golf Magazines' Representations of Disability, Gender, and Age», Sociology of Sport Journal, vol. 18 issue 1, pp. 21-36.

MORENO, I. (2003): Narrativa audiovisual publicitaria. Barcelona, Paidós.

PANIZA PRADOS, J.L. (2005): La percepción social del golf en Andalucía. Sevilla. Consejería de Turismo, Comercio y Deporte.

PETRICK, J. F. (2002): «Experience use history as a segmentation tool to examine golf travellers satisfaction, perceived value and repurchase intentions», Journal of Vacation Marketing, 8 (4), pp. 332-342.

PRIESLEY, G. y SABI, J. (1987): «The role of golf as tourist attraction: the case of Catalonia Spain», Acts of the Meeting of the Commission of Geography of Tourism, Leisure and recreation of the International Geographical Union. Sousse. Tunisia.

RIQUEL LIGERO, F. y VARGAS SÁNCHEZ, A. (2012): «El entorno institucional de carácter medioambiental de los campos de golf andaluces: un análisis factorial», Cuadernos de Turismo, n 29, pp. 209-229.

SANTIBÁÑEZ, A. Y VERGARA, E. (2008): «Periodismo y Publicidad: claves y ambigüedades de una relación promiscua», Revista Universum, n 23 , vol. 1, pp. 248-267.

SCHLÜTER, R. (2003): El turismo en Argentina. Del balneario al campo . Buenos Aires, Centro de Investigaciones y Estudios Turísticos.

TROITIÑO VINUESA, M.A. (2002): «Turismo y recuperación urbana», en El turismo en Castilla-La Mancha. Análisis y perspectivas. Cuenca, Universidad de Castilla-La Mancha, pp. 29-43. 
WILSON, J. Y THILMANY, D. (2006): «Golfers in Colorado: The Role of Golf in Recreational and Tourism Lifestyles and Expenditures», Journal of Travel \& Tourism Marketing, Vol. 20 Issue 3/4, pp. 127-144. 